Pressure Vessel Design and Analysis 


\section{Pressure Vessel \\ Design and Analysis}

\section{B. BICKELL M.SC.}

Contract Manager, Babcock \& Wilcox Ltd.

\section{RUIZ DR.ING}

Senior Lecturer in Mechanical Engineering, University of Sheffield

Macmillan Education 
(C) M. B. Bickell and C. Ruiz 1967

Softcover reprint of the hardcover 1st edition 1967

MACMILLAN AND COMPANY LIMITED

Little Essex Street London WC2

also Bombay Calcutta Madras Melbourne

THE MACMILLAN COMPANY OF CANADA LIMTTED

70 Bond Street Toronto 2

ST. MARTIN'S PRESS INC 175 Fifth Avenue New York NY 10010

First Published 1967

$9^{\prime \prime} \times 6^{\prime \prime}, 592$ pages 198 line illustrations

Library of Congress Catalog Card Number 67-20870

ISBN 978-1-349-00131-6

ISBN 978-1-349-00129-3 (eBook)

DOI 10.1007/978-1-349-00129-3 


\section{Contents}

Introduction $\quad \mathrm{ix}$

Notation $\quad \mathrm{xi}$

1 Design Procedure and Establishment of the Design Requirements

Introduction. 1 Functional Requirements. 1.1 Size and Shape. 1.2 Fluid Contained. 1.3 Method of Support. 1.4 Location of Attachments and Penetrations. 2 Operational Requirements and Limitations. 2.1 Maintained Loading. 2.2 Transient Conditions. 2.3 Severity of Duty. 3 Principal Design Codes. 3.1 Safety Devices. 3.2 Pressure Testing.

2 Selection of Materials

Introduction. 1 Steel. 1.1 Carbon Steel. 1.2 Low Alloy Steels. 1.3 High Alloy Steels. 1.4 Clad Steels. 2 Non-ferrous Metals. 2.1 Aluminium and Aluminium Alloys. 2.2 Copper and Copper Alloys. 2.3 Nickel and Nickel Alloys.

3 Preliminary Layout

Introduction. 1 Establishment of the Design Conditions. 2 Nominal Design Stress. 3 Basic Shell Thickness. 3.1 Cylinders and Spheres under Internal Pressure. 3.2 Cylinders and Spheres under External Pressure. 4 Dimensioning of Local Components. 4.1 Heads. 4.2 Reinforcement of Openings. 4.3 Special Components (Flanges, Tube Plates, Supports). 5 Manufacture. 5.1 Manufacturing Tolerances. 5.2 Welding Processes and Inspection Techniques. 5.3 Design of Welded Joints. 5.4 Butt Welding of Plates of Unequal Thickness. 5.5 Head to Shell Connections. 5.6 Welded Nozzles. 5.7 Welded Attachments. 6 Stress Relieving.

4 Elastic Stress Analysis: General Method

Introduction. 1 Stresses, Strains and Displacements. 2 Assumptions of Thin Shell Theory. 3 Membrane and Bending Behaviour of Shells. 4 The Use of Matrices. 5 Axi-symmetric Loading. 5.1 Co-axial Shells Joined in Series. 5.2 Co-axial Shells which Intersect in a Common Circle. 6 Lateral Loading. 6.1 Co-axial Shells Joined in Series. 6.2 Co-axial Shells which Intersect in a Common Circle. 7 General Loading.

5 Cylindrical Shells

Introduction. 1 Thick Cylinders. 1.1 Finite Difference Methods for Axisymmetric Loading. 2 The Basic Equations for Thin Cylindrical Shells. 3 Axi-symmetric Loading. 3.1 Membrane Solutions. 3.2 Edge bending Solutions and the Flexibility Matrix. 3.3 Unrestrained Solutions. 3.4 Cylinders of Variable Thickness. 4 Lateral Loading. 4.1 Membrane Solutions. 4.2 Edge bending Solutions and the Flexibility Matrix. 4.3 Unrestrained Solutions and Applications. 5 General Loading. 
Introduction. 1 Thick Spheres. 2 The Basic Equations for Thin Spherical Shells. 3 Axi-symmetric Loading. 3.1 Membrane Solutions. 3.2 Edge bending Solutions and the Flexibility Matrix. 3.3 Unrestrained Solutions and Applications. 4 Lateral Loading. 4.1 Membrane Solution. 4.2 Edge bending Solutions and the Flexibility Matrix. 4.3 Unrestrained Solution and Applications. 5 General Loading.

\section{General Shells of Revolution}

Introduction. 1 Thick Shells. 2 The Basic Equations for Thin Shells. 3 Axisymmetric Loading. 3.1 Membrane Solutions. 3.2 Edge bending Solution and the Flexibility Matrix. 3.3 Surface Loads and Temperature Gradients. 3.4 Numerical Methods of Solution. 4 Flat Annular Plates., 5 Conical Shells. 5.1 Conical Shells with Variable Thickness. 6 Ellipsoidal Shells. 7 Toroidal Shells.

\section{Application of the General Analysis to the More Usual Vessel} Components

Introduction. 1 Flat Closure Plates. 2 Conical Heads and Reducers. $3 \mathrm{Hemi}-$ spherical Heads. 4 Torispherical and Ellipsoidal Heads (Dished or Domed ends). 5 Tapered Transition Joints. 6 Reinforcement of Openings. 6.1 Single Radial Nozzles: Internal Pressure. 6.2 Single Oblique Nozzles: Internal Pressure. 6.3 Multiple Openings. 6.4 Flued Openings and Flared Nozzles. 7 Special Methods for the Design of Reinforced Openings.

9 Stress Analysis of Piping Systems

Introduction. 1 Single Run of Pipe. 2 Elements of the Flexibility Matrix. 2.1 General Plane Curve. 2.2 Straight Length of Pipe of Constant Circular Cross-Section. 2.3 Bend with Constant Circular Cross-Section following a Circular Arc. 3 Unrestrained Displacements-Thermal Loads. 4 Unrestrained Displacements-Mechanical Loads. 5 Multi-anchor Runs of Pipe.

10 Adequacy of the Design: General Considerations

Introduction. 1 Development of Cracks: Fracture. 2 Corrosion. 3 Working Stresses. 3.1 Field of Application of the Elastic Method of Stress Analysis. 3.2 Experimental Stress Analysis. 3.3 Selection of Working Stresses for Ductile Materials. 3.4 Brittle Materials. 3.5 Summary.

\section{Plastic Collapse}

Introduction. 1 Bursting Pressure of Thin-Walled Spherical and Cylindrical Vessels. 2 Bursting Pressure of Thick-Walled Spherical and Cylindrical Vessels. 2.1 Spherical Vessels. 2.2 Cylindrical Vessels. 2.3 Cylindrical Vessels: Anisotropic, Strain Hardening Materials. 2.4 Approximate Expressions. $2.5 \mathrm{Com}-$ pound Cylindrical Vessels. 3 Tresca Yield Criterion for Rigid/Ideally Plastic Shells. 3.1 Exact Condition. 3.2 Simplified Yield Conditions. 4 Application of Limit Analysis to Design. 4.1 General Principles. 4.2 Simply-supported Circular Plate under Uniform Load. 4.3 Band Reinforced Cylindrical Vessel. 4.4 Collapse Loads of Several Shells of Revolution.

12 Fatigue and Incremental Collapse

Introduction. 1 Incremental Collapse. 2 Fatigue of Pressure Vessel Materials. 2.1 Cumulative Fatigue Damage. 2.2 Effect of Notch-Type Stress Raisers. 2.3 Effect of Other Variables. 3 Application to Design. 
13 Low Stress Brittle Fracture

Introduction. 1 Interpretation of the Experimental Information. 1.1 The Classical Theory. 1.2 Griffith's Theory of Crack Propagation. 2 Experimental Determination of the Transition Temperature: Toughness Tests. 2.1 Correlation between Test Results. 2.2 State of Stress at Notches and Size Effect. 2.3 Correlation with-Service Experience. 3 Application to Design. 3.1 General Considerations. 3.2 Application of Toughness Tests.

\section{Creep}

Introduction. 1 Presentation and Availability of Data. 2 Extrapolation of Data. 3 Interpretation of Data: Uni-axial Stress Systems. 3.1 The Superposition Method: Steady Creep and Creep Recovery. 3.2 Strain and Time Hardening Theories: Creep Damage Factor. 3.3 Stress Relaxation. 4 General Stress-Strain Relations. 5 Applications. 5.1 Thick-Walled Cylindrical and Spherical Vessels under Internal Pressure. 5.2 Steady Creep of Thin Cylindrical Vessels under Internal Pressure and Axial Loading. 5.3 Steady Creep of Thin Cylindrical Vessels under Internal Pressure and Bending. 5.4 Steady Creep of Thin Cylindrical Vessels under Internal Pressure and Torsion. 5.5 Piping Systems and Shells of Revolution. 6 Design Stress in the Creep Range. 6.1 High Strain Fatigue combined with Creep. 6.2 Effect of Local Constraints, Discontinuity Stresses and Notch-Type Stress Raisers.

15 Buckling

Introduction. 1 Cylindrical Vessels under Uniform External Pressure. 1.1 Critical Buckling Pressure. 1.2 Effect of Initial Out-of-Roundness and Plastic Collapse. 1.3 Stiffening Rings. 1.4 Design Procedure. 2 Spherical Vessels under Uniform External Pressure; Dished Ends. 3 Effect of End Supports and Structural Discontinuities.

16 Special Components

Introduction. 1 Smooth Bends. 1.1 Flexibility Factor of Smooth Bends. 1.2 Stress Distribution: Pressure and External Loading. 1.3 Design. 2 Mitred Bends and Lobster-Back Bends. 3 Tube Plates. 3.1 Effective Elastic Constants. 3.2 Stress Distribution in the Equivalent Plate. 3.3 Stress Analysis. 3.4 Peak Stresses. 3.5 Stress Limits: Design. 4 Bolted Flanged Connections. 4.1 Steady Creep of Bolted Flanged Connections.

Note on Recent Bibliography 


\section{Introduction}

The traditional method for the design of pressure vessels is characterized by the existence of Design Codes. These are based on the application of an arbitrary factor on the ultimate tensile strength or yield point of the material used and include rules, derived from previous experience, for the design of certain local components such as branches and supports. The primary design criterion implicit in these rules is the prevention of bursting or buckling under uniform pressure loading. Although this is sufficient to ensure the integrity under initial loading and the safe operation of most vessels it does not always lead to an economic and safe design; indeed under certain operating conditions, such as rapid changes in temperature or pressure, a vessel designed according to Code rules may be unsafe. Several Code writing authorities have recognized this situation and some of the most recently published Codes permit more economic design and at the same time require a detailed study of the possibility of failure.

The starting point for such a study is a complete specification of the service conditions followed by an analysis of the possible modes of failure. Since the most common modes of failure are stress dependent, the determination of the stresses and the establishment of acceptable limits are essential steps in the design process. Pressure vessels are critical structures and the risks involved in their operation are often high. As a result, new theories and methods of analysis can only be applied to design when they become sufficiently well established to be generally accepted. On the other hand, designers should be aware of any developments in order to apply them at the first opportunity. We have therefore endeavoured to include the basic information which we believe the designer should use on the methods of stress analysis and the principal modes of failure. At the same time we have briefly discussed and quoted references to more specialized and advanced work which has not yet been widely used in design.

The first step in the design process is the establishment of a preliminary layout. This is dealt with in Chapters $1-3$, which contain references to the appropriate Design Codes and to sources of information on materials properties. The next step is usually an elastic stress analysis following the general methods described in Chapter 4 using the detailed results from Chapters 5-7. 
We believe there are significant advantages in developing the theory of shells in a manner which is specifically directed towards the type of problems which arise in pressure vessel design. With this approach it is possible to concentrate on certain aspects of the theory and to carry these forward to a stage which reduces the work required to apply the text results to actual problems. In addition we have attempted to clarify the unified nature of the theory of axisymmetric shells by adopting the same chapter sub-divisions for the different shapes considered. To obtain the full benefit of these chapters the reader will need to study them and will require a mathematical knowledge to University engineering graduate standard. Designers lacking this knowledge will still be able to apply the results of some of this information which are summarized in Chapter 8. For completeness the stress analysis of piping systems has been included in Chapter 9.

In addition to the elastic stress analysis, an essential part in the design process is the establishment of acceptable working stresses and, when possible, the determination of the maximum load that the vessel may resist without failure. A general introduction to this problem is provided by Chapter 10, which contains also a brief discussion of stress-independent modes of failure. In the following chapters an attempt has been made to describe the application of experimental data, obtained from simple mechanical tests, to the design of complex vessels. Supplementary design rules based on experience are included also.

In recent years limit analysis has received considerable attention, since it has the advantage of providing a means for the direct evaluation of the load carrying capacity of the structure. Chapter 11 describes the fundamental aspects together with some simple examples and several results.

In a final chapter the principles discussed are applied to the design of some special components. These are pipe bends, tube plates and bolted connections. As in Chapter 8, the results are presented in a simplified form which may be applied without demanding more than an elementary knowledge of mathematics.

Most of the information contained in this book was collected or developed whilst both authors were employed by Babcock \& Wilcox Ltd. to whom thanks are extended both for support during the preparation of the book and for permission to publish. We are also grateful for the advice and encouragement given by many of our friends and colleagues. 


\section{Notation}

$A$ Area

$\alpha$ Linear thermal expansion coefficient

A Flexibility matrix

B Stiffness matrix

$\beta \quad$ Flexibility characteristic

$D$ Flexural rigidity, diameter

d Diameter

$D^{*} \quad$ Equivalent flexural rigidity of perforated plate

$\delta$ Deflection

D Generalized displacement matrix

$E$ Modulus of elasticity

$E^{*}$ Equivalent modulus of elasticity of perforated plates

E Strain vector

$\dot{\mathbf{E}}$ Strain rate vector

$\varepsilon$ Strain

$\varepsilon_{F} \quad$ Logarithmic strain at rupture $=\log _{e} \frac{100}{100-R A}$

$\bar{\varepsilon} \quad$ Significant strain

$\dot{\varepsilon}$ Strain rate

E.S.R. Equivalent stress range

$F$ Force

F Generalized force

$h$ Shell thickness, height of nozzle reinforcement

$J$ Joint efficiency factor

I Moment of inertia

I Identity matrix

$K_{f}, K_{t}$ Effective and theoretical stress concentration factors

$K_{F} \quad$ Flexibility factor of pipe bend

$k_{1}, k_{2}$, Flexibility factors of pipe subjected to in-plane and out-of-plane

$k_{3} \quad$ bending moments and torque

$L, l$ Length

$\lambda$ Pipe factor 
$M$ Bending moment, resultant bending moment

$m$ Dimensionless bending moment

$N$ Number of cycles, resultant force normal to shell thickness

$n$ Dimensionless resultant force

$v$ Poisson's ratio

$v^{*}$ Equivalent Poisson's ratio of perforated plates

O Null matrix

$P$ Pressure

$p$ Dimensionless pressure

$Q$ Resultant shear

$q$ Reduced shear

$R, r$ Shell radius

$R A$ Reduction of area at fracture (tensile test)

$S_{a} \quad$ Allowable or design stress

$S_{e}$ Endurance limit

S Stress vector

$\sigma$ Stress

$\bar{\sigma}$ Significant or combined uni-axial stress equivalent to a general stress system

$S$ Stress concentration factor for a pipe bend (bending stress)

S.F. Safety factor

S.C.F. Stress concentration factor

$T$ Temperature, shell thickness

$t$ Shell thickness

$\theta$ Time

$\theta_{r}$ Time-to-rupture (creep)

$\tau$ Shear stress

U.T.S. Ultimate tensile strength

$u, v, w$ Components of displacement

$V$ Deformation rate in yielded shell

$W$ Strain energy, work or rate of dissipation of energy

$Y$ Yield point stress

$\chi$ Tangent rotation, change in curvature

\section{Subindices}

1, 2, 3 Applied to stress or strain refer to the principal components

$x, y, z, \phi, \theta$ Applied to stress, force, deflection or rotation refer to the components in the relevant directions

$o, i$ Applied to radius or pressure to respective magnitudes at the outside or inside surfaces of a shell

$e, p, c$ Refer to elastic, plastic or creep components of stress, etc. 